\title{
SELECCION SOCIAL E INGRESO A LA UNIVERSIDAD PÚBLICA: Universidad del Valle ${ }^{\star}$
}

Fabio E. Velásquez $C$.*

\section{Introducción}

Los análisis sobre la Universidad y, en general, de la educación superior, parecen aceptar cada vez más que el acceso de los jóvenes a los estudios profesionales supone un proceso de selección social que opera fundamentalmente, aunque no de manera exclusiva, a partir del origen social y adquiere rasgos progresivamente explícitos en las sucesivas fases del ciclo educativo. En su conocido estudio sobre los universitarios franceses, Bourdieu y Passeron señalan que "la desigualdad inicial de los diferentes estratos sociales ante la escuela se manifiesta, en primer lugar, por el hecho de estar muy desigualmente representados en ella" ${ }^{1}$, de modo que las capas sociales más representadas en la enseñanza superior son, al mismo tiempo, las que menos peso proporcional poseen en la población activa. Tal desigualdad se expresa en formas muy diversas: los alumnos de clases inferiores tienden a ser relegados a ciertas disciplinas y muestran estancamiento en los estudios: su capacidad de elección parece ser más restringida y su bagaje cultural los coloca en una situación desfavorable con respecto a sus colegas de clase alta pues "para los individuos de los estratos menos favorecidos la escuela sigue siendo la única vía de acceso a la cultura en todos los niveles de escolaridad"2.

En consecuencia, la igualdad aparente de los estudiantes universitarios en el sentido de constituir una población sometida a prácticas y rituales similares (ir a clase, recibir cursos, preparar y presentar evaluaciones, establecer una relación social con los profesores, desarrollar actividades gremiales y académicas conjuntamente, etc.) es tan sólo una igualdad formal que esconde una serie de diferencias engendradas por su diverso origen social. En tal sentido, carece de validez la hipótesis de la "meritocracia" según la cual la llegada a la Universidad es el fruto del despliegue de capacidades y méritos individuales en un contexto de igualdad de oportunidades. No sólo esa "igualdad de oportunidades" es formal sino que, si bien existen capacidades y habilidades distintas entre los pretendientes a la educación superior, ellas no operan y no se entienden por fuera de la relación con los saberes y predisposiciones que asigna el origen social a cada individuo. Más aún, el hecho de que no todos los que aspiran pueden obtener un cupo en la Universidad no significa que el examen de admisión se convierta en el juez máximo sino que, como lo señalaron Bourdieu y Passeron, lo que se lleva a cabo es una "elección de los elegidos": "Es tal la eficacia de los factores sociales de diferenciación que, aun cuando se consiguiera la igualdad en el aspecto económico, el sistema universitario seguiría consagrando las desigualdades mediante la transformación de los privilegios sociales en dotes o méritos individuales. Más aún, una vez conseguida la igualdad formal de probabilidades, la escuela podría ofrecer la apariencia de la más completa legalidad al servicio de la legitimación de los privilegios" ${ }^{3}$.

\footnotetext{
"Ponencia presentada al IV Congreso Nacional de Sociología. Cali, agosto 4-6 de 1982.

** Profesor auxiliar. Departamento de Ciencias Sociales, Universidad del Valle, Cali.

${ }^{1}$ Bourdieu, Pierre y Passeron, J.C., Los estudiantes y la cultura, Nueva Colección Labor, 2a. Ed., Barcelona, 1969. p. 25.

${ }^{2}$ Ibid., p. 47.

${ }^{3}$ Ibid., p. 54. 
La investigación de Baudelot y Establet sobre la escuela capitalista en Francia ${ }^{4}$, aunque crítica frente a algunos planteamientos de Bourdieu y Passeron, se ubica en la misma línea de análisis. Muestran, en efecto, los autores la existencia de lo que denominan "dos redes de escolarización" diferentes que tienen su punto de partida en la primaria y se desarrollan de manera "independiente" a lo largo de la educación escolar. Esas redes, que poseen cada una sus propias prácticas pedagógicas y su lógica de organización y funcionamiento, acogen de manera diferenciada a estudiantes de distinto origen social y contribuyen por esa vía a la reproducción de las relaciones sociales de producción mediante la formación, por una de ellas, de trabajadores manuales y, por la otra, de trabajadores intelectuales, y mediante la transmisión de la ideología burguesa:

“¿Cómo se asegura la reproducción de las relaciones de producción, en lo que le concierne, en el aparato escolar? Mediante los efectos de su mecanismo único que produce:

1)La repartición material de los individuos en dos masas desiguales $(75 \%-25 \%)$ distribuidas en dos redes internas de la escuela y entre dos posiciones tendencialmente opuestas de la división del trabajo sobre las cuales estas dos redes desembocan en el exterior de la escuela;

2)La inculcación de la misma ideología burguesa bajo dos formas diferentes, correspondientes a las dos masas consideradas, con un destino único: el mantenimiento de las relaciones de producción existentes. Esta vinculación va a la par, como hemos visto, con el rechazo y el disfraz de la ideología del proletariado"5.

En lo que respecta a la universidad colombiana, la hipótesis de la selección social ha guiado igualmente varios estudios. Cataño asegura que "a la universidad (...) ingresan solamente aquellos estudiantes que han triunfado sobre los obstáculos presentados por los ciclos primario y secundario. Estos obstáculos, según se ha tratado de mostrar, son sobre todo de carácter social y económico, presentándose el hecho de que los niveles inferiores de la estratificación social poseen, frente a los otros, menos chances de obtener

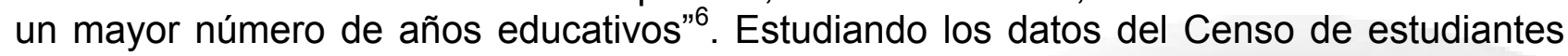
de la Universidad Nacional de 1967, muestra cómo la mayoría de las variables analizadas (ambiente educativo familiar, origen escolar, origen ecológico, origen social) llevan a la conclusión de que el grueso de la población universitaria procede de la clase media, de los "estratos medios medios y medios altos"; para ellos la Universidad se convierte en un elemento de prestigio y, ante todo, en "un medio de retener la posición social, heredada del padre"7. Por esa vía, Cataño arriba a la siguiente conclusión: "la estratificación social de la Universidad Nacional nos muestra también cómo ella reproduce la estratificación social de la sociedad pero de manera invertida. Las clases sociales más representadas en la Universidad son las menos representadas en la población activa" ${ }^{8}$.

El análisis de Rama sobre el sistema universitario colombiano a finales de la década del $60^{9}$ señala que:

\footnotetext{
${ }^{4}$ Baudelot, Chistian, y Establet, Roger. La Escuela Capitalista, México, siglo XXI, 1975.

5 lbid., p. 254. (subrayado de los autores).

6 "Universidad Pública y Movilidad Social", en UN, Revista de la Dirección de Gataño, Gonzalo, Divulgación Cultural, Universidad Nacional de Colombia, No. 5, Bogotá, Septiembre de 1970, p. 198.

7 Ibid., p. 222.

8 lbid., p. 223.

${ }^{9}$ Rama, Germán, El Sistema Universitario en Colombia, Universidad Nacional de Colombia, Bogotá, 1970.
} 
"La Universidad es la imagen invertida de la sociedad, ya que las categorías socioocupacionales de mayor volumen dentro de la sociedad -caso de los asalariados manuales - no tienen más que una representación insignificante de la matrícula universitaria, mientras que categorías cuantitativamente muy poco significativas, como son los profesionales universitarios, los empleados de calificación técnica y los jefes altos o intermedios de la administración pública o privada (...) que apenas suman el $4 \%$ de la población económicamente activa, retienen el $27.5 \%$ de las plazas de la Universidad Nacional y el $46.4 \%$ de las plazas de la Universidad de Los Andes" $"$.

Esta afirmación, que, al igual que la de Cataño, se encuentra muy a tono con el enfoque de Bourdieu y Passeron sin que necesariamente se inspire teóricamente en ellos, es complementada por otras similares. Analizando, por ejemplo, las características educativas de los estudiantes universitarios afirma: "El proceso de selección iniciado en la escuela primaria se continúa a lo largo del ciclo secundario a favor de los estudiantes que asisten a instituciones privadas"11.

En su estudio sobre el origen social y el rendimiento académico de los estudiantes de las Universidades Nacional y de los Andes, Lucía Tarazona acoge también el enfoque de la selección social. Establece desde el comienzo que "el proceso educativo puede analizarse como un proceso de selección social por el cual todos o ciertos individuos y grupos participan —en diversos grados - de los bienes sociales y culturales que implica la escolarización, que son bienes requeridos para el desempeño de ciertos roles sociales" ${ }^{12}$. De esa manera, la composición social de la Universidad pública y el rendimiento académico de sus estudiantes se hallan determinados por su origen social específico, en contraste con lo que ocurre en la universidad privada:

"Verdaderamente, afirma Tarazona, una situación como ésta está mostrando cómo opera socialmente un criterio académico severamente selectivo (...) En efecto, si en ninguna de las universidades se ha borrado el efecto de las desigualdades de origen social sobre los logros académicos, en la Nacional un criterio académico estricto redunda en mayores oportunidades de éxito académico para las categorías sociales inferiores, en tanto que en Los Andes, su concepto altamente selectivo se traduce en mínimas oportunidades de éxito académico para las categorías ocupacionales inferiores, en relación con las demás categorías sociales"13.

En un trabajo un poco más reciente, Parra ${ }^{14}$ califica de "mito" a la hipótesis de la educación como factor de movilidad social y muestra cómo esa idea se institucionalizó en Colombia desde que se abrió paso el proceso de modernización educativa en los años cuarenta de este siglo, marcando un cambio en cuanto al volumen, tipos de ramas del conocimiento, y contenidos educativos, en cuanto al desarrollo de políticas de construcción de escuelas y de formación de maestros, y en su conjunto en cuanto a la función que dentro de la sociedad colombiana cumple la educación" ${ }^{15}$. Por el contrario, afirma Parra,

\footnotetext{
10 lbid., p. 83.

11 lbid., p. 83.

12 Tarazona, Lucía, Origen Social y Rendimiento Académico de los Estudiantes en la Universidad Pública y en la Universidad Privada, (Trabajo presentado como requisito para optar el título de Licenciatura en Sociología), Universidad Nacional, Bogotá, 1969, p. 2.

13 lbid., p. 103.

14 Parra, Rodrigo, Análisis de un mito: la educación como factor de la movilidad social en Colombia, Universidad de los Andes, Bogotá, 1973.

${ }^{15}$ lbid., p. 121.
} 
en la realidad ocurre algo muy diferente, pues todo indica que históricamente la educación ha servido de vehículo de implementación de elementos de diverso orden que han contribuido a consolidar, en vez de quebrantar, la rigidez de la estructura social. Por ello,

\begin{abstract}
"cuando se mira con un poco de detenimiento la sociedad colombiana en lo que se refiere a la movilidad social se observa que: a) las posibilidades de movilidad ascendente que ofrece dentro o fuera del canal educativo son mínimas; b) esas posibilidades de movilidad por medio del canal educativo sólo existen para grupos específicos, estratos medios y altos urbanos, para los que realmente y en términos generales no representa un ascenso sino un elemento de mantenimiento de la posición; c) el fenómeno más importante y masivo es el de movilidad descendente, por medio de la proletarización rural y la marginalización de grandes masas"16.
\end{abstract}

Así, pues, las tesis de la selección social de los estudiantes universitarios tiene una amplia acogida entre los estudiosos de la educación superior, independientemente de la postura teórica que adoptan y de los distintos matices que adquiere en la pluma de cada uno de ellos. Es indudable que dicha tesis posee suficientes fundamentos teóricos y empíricos y resulta, por ello mismo, muy útil para el estudio de casos concretos. Sin embargo, como hipótesis que es, constituye una directriz general para el análisis pero nada dice, ni puede decir, sobre la especificidad de esos casos, vale decir sobre los mecanismos mediante los cuales opera y sobre su alcance en una sociedad determinada.

En realidad, lo que interesa para el análisis no es tanto silos procesos de selección tienen o no ocurrencia real, sino la manera concreta como se desarrollan y las formas específicas que adoptan en un lugar y un tiempo determinados. Corresponde al análisis sociológico no tanto establecer leyes generales de validez universal, que por su elevado nivel de abstracción se encuentran vacías de contenidos históricos, sin o precisar la especificidad histórica de la materia que trata y los factores que la configuran como una individualidad históricamente significativa. Partiendo de este supuesto metodológico, las interpretaciones expuestas en este trabajo tienen la pretensión de examinar los procesos de selección social relacionados con el ingreso de bachilleres a una universidad pública, la Universidad del Valle, mostrar los canales a través de los cuales operan y precisar su influencia sobre el papel social de la institución universitaria.

Trataré de mostrar cómo la entrada a la universidad puede y debe ser mirada como una difícil competencia en la que un alto volumen de aspirantes participa en condiciones aparentemente iguales pero realmente inequitativas. Esa desigualdad, de carácter esencialmente social, está íntimamente relacionada con las transformaciones recientes del sistema universitario colombiano, lo mismo que con la rápida masificación de la enseñanza media y su efecto más inmediato, el acceso de sectores populares y de capas medias asalariadas a la educación.

La información en que se funda el análisis ha sido tomada de un estudio que viene realizando un grupo de investigadores de la Universidad del Valle sobre los aspirantes y los estudiantes de la Universidad y del cual formo parte ${ }^{17}$. Aunque algunas líneas

\footnotetext{
${ }^{16}$ Idem.

17 El equipo está conformado por los sociólogos Lugardo Alvarez y Fabio Velásquez y los Licenciados Américo Calero y Lida C. de Bolaños. La investigación se titula Aspirantes, estudiantes y egresados: el caso de la Universidad del Valle y viene siendo realizada con el apoyo del Centro de Investigaciones y Documentación Socio-Económica (CIDSE), el Departamento de Curriculum (División de Educación) y la Oficina de Planeación y Desarrollo de la Universidad del Valle.
} 
generales del análisis han sido comentadas en el equipo, debe quedar muy claro que las afirmaciones contenidas en estas páginas son hechas bajo mi exclusiva responsabilidad.

La exposición ha sido dividida en dos grandes bloques: en el primero, hago un estudio de las formas específicas que asume el proceso de selección de aspirantes y estudiantes; en el segundo, examino el problema de las preferencias académicas y propongo algunos puntos de vista sobre las tesis de la Universidad como canal de reproducción social.

\section{Una competencia ardua y desigual}

Para nadie es un secreto que entrar a la Universidad del Valle se ha vuelto una meta cada vez más difícil de lograr. En el Valle del Cauca, los niveles de satisfacción de la demanda de plazas universitarias han disminuido sensiblemente en la última década. En 1971, los establecimientos de enseñanza universitaria absorbieron el $23.1 \%$ de los aspirantes, mientras que en 1978 , tan sólo un $30.2 \%$. La competencia por cupos, que no es sino la otra cara de la dificultad, ha sido mayor en los establecimientos oficiales. En 1971, por cada cupo ofrecido por estos últimos había un promedio de 2.2 aspirantes, mientras que solamente había 1.6 aspirantes por cupo en las universidades privadas. En 1978 , esas cifras eran, respectivamente de 5.4 y 2.4 aspirantes por cupo.

La Universidad del Valle, en razón de su importancia regional y nacional y de su carácter de establecimiento público, refleja de manera típica esa tendencia: en 1971, competía por cada cupo ofrecido 2.7 aspirantes; en 1975, lo hacían 3.7 y en 1981, 5.3 aspirantes. No es complicado precisar los factores más significativos que explican ese fenómeno. En primer lugar, el volumen cada vez mayor de bachilleres egresados de la educación media en sus diversas modalidades. La matrícula en último año de bachillerato en el Valle del Cauca, región de influencia más importante de la Universidad ${ }^{18}$ ha venido creciendo rápida, casi que vertiginosamente, al punto de haberse doblado en sólo seis años (Ver Cuadro 1). El acceso progresivo de nuevos grupos sociales, particularmente de sectores populares, a la educación secundaria, en virtud no solamente de una "socialización" de la educación media por la vía de la ampliación de cupos, sino también de las exigencias cada vez más severas de calificación en el mercado laboral, ha producido una mayor demanda y, en consecuencia, una mayor presión sobre los cupos en la educación superior, particularmente la universidad.

Frente a esta creciente demanda, la Universidad del Valle viene ofreciendo, en segundo lugar, un número de plazas cuyo crecimiento es inferior al del número de bachilleres y aspirantes. Como lo indican claramente las cifras del Cuadro 1, mientras que la matrícula en último año de bachillerato en el Valle del Cauca se duplicó entre 1972 y 1978, el volumen de aspirantes se triplicó en el mismo período, al tiempo que el número de cupos ofrecidos por la Universidad ni siquiera se alcanzó a duplicar. Más aún, la tendencia muestra una disminución progresiva del número de plazas ofrecidas por la Universidad, lo mismo que de su matrícula en primer año. Esto último ha obedecido, de una parte, al "filtro" impuesto por la Universidad a la población de aspirantes, representado en la exigencia de un puntaje mínimo en los exámenes de ICFES para poder inscribir-se en las pruebas de admisión; de otra, a la angustiosa situación financiera que viene experimentando la institución desde hace cinco o seis años, la cual le impide, entre otras

\footnotetext{
${ }^{18}$ La gran mayoría de aspirantes de la Universidad del Valle (cerca del 90\% en 1980 y 1981) terminaron su secundaria en establecimientos localizados en el Valle del Cauca. El resto, provienen principalmente del Cauca, Nariño, Risaralda y Cundinamarca. Es muy baja la representación de otras zonas del país.
} 
cosas, y a pesar de políticas de ampliación de cupos del gobierno nacional, atender a un número mayor de estudiantes.

Se configura, así, una situación de déficit de cupos que, por sí misma, se convierte en un primer mecanismo de selección social de los estudiantes: no todos aquellos que están en capacidad ${ }^{19}$ de cursar estudios universitarios pueden hacerlo, pues el hecho de finalizar la educación media no les garantiza de por sí su entrada a la Universidad. La existencia de la Universidad privada, si bien es cierto que amplía la oferta, disminuyendo aparentemente el déficit, en realidad, en lugar de aliviar el problema, lo agra-va pues su rasgo principal es el de los altos niveles de matrícula, que contribuyen a hacer aún más selectivo el ingreso a la Universidad, como se mostrará más adelante, lo que hace la Universidad privada es ampliar el abanico de alternativas para aquel sector de estudiantes que, por provenir de familias de altos ingresos, no encuentran obstáculos para pagar un alto precio por sus estudios profesionales. Quienes no pueden hacerlo, deben competir para lograr su puesto en un establecimiento oficial. A ello hay que añadir que la Universidad del Valle garantiza un buen nivel académico en sus planes de estudio, mejor que el ofrecido por la mayoría de los centros privados, lo cual aumenta la presión sobre sus cupos sin que pueda satisfacer totalmente la demanda ${ }^{20}$.

\section{CUADRO 1}

\begin{tabular}{|c|c|c|c|c|}
\hline \multicolumn{5}{|c|}{$\begin{array}{l}\text { MATRICULA EN ULTIMO AÑO DE BACHILLERATO EN EL VALLE DEL CAUCA, ASPIRANTES, } \\
\text { CUPOS Y MATRICULA EN PRIMER AÑO EN LA UNIVERSIDAD DEL VALLE, } 1971 \text { - } 1980 \text { (INDICES) }\end{array}$} \\
\hline Años & $\begin{array}{l}\text { Matrícula en último } \\
\text { Año de bachillerato } \\
(1971-72=100)\end{array}$ & $\begin{array}{c}\text { Aspirantes } \\
\text { a la } \\
\text { Universidad } \\
\text { del valle } \\
(1972=100)\end{array}$ & $\begin{array}{c}\text { Cupos en la Universidad } \\
\text { Del valle } \\
(1972=100)\end{array}$ & $\begin{array}{c}\text { Matrícula en } \\
\text { Primer año U.V } \\
(1972=100)\end{array}$ \\
\hline 1971-72 & 100.0 & 100.0 & 100.0 & 100.0 \\
\hline \multicolumn{5}{|l|}{1972} \\
\hline $1972-73$ & 114.9 & 105.5 & 86.3 & 71.3 \\
\hline \multicolumn{5}{|l|}{1973} \\
\hline $1973-74$ & 123.4 & 137.3 & 103.2 & 96.7 \\
\hline \multicolumn{5}{|l|}{1974} \\
\hline 1974-75 & 151.5 & 181.9 & 129.8 & 135.4 \\
\hline \multicolumn{5}{|l|}{1975} \\
\hline $1975-76$ & 184.4 & 253.4 & 191.9 & 177.4 \\
\hline \multicolumn{5}{|l|}{1976} \\
\hline 1976-77 & 211.1 & 334.7 & 164.0 & 149.1 \\
\hline \multicolumn{5}{|l|}{1977} \\
\hline $1977-78$ & 205.5 & 164.0 & 140.0 & 172.1 \\
\hline 1978 & & & & \\
\hline 1978-79 & S.I. & 197.3 & 135.4 & (a) \\
\hline \multicolumn{5}{|l|}{$1979-80$} \\
\hline 1980 & S.I. & 242.5 & 115.6 & 144.2 \\
\hline
\end{tabular}

Fuentes: Anuarios Estadísticos del Valle del Cauca. Oficina de Planeación y Desarrollo - Univalle.

\footnotetext{
${ }^{19}$ Nótese que se habla de quienes "están en capacidad" y no de quienes "están en edad" de ingresar a la Universidad. En verdad, la educación primaria y secundaria, mediante mecanismos educativos y extraeducativos (sociales), llega a ser el primer instrumento de selección de los estudiantes universitarios en la medida en que "deja tirados" en el camino a muchos jóvenes que no culminan el bachillerato.

${ }^{20}$ En 1978, la Universidad del Valle atendió el $45.9 \%$ de las solicitudes de ingreso a la educación superior y el $50.7 \%$ de las solicitudes a estudios universitarios. Le siguieron la Universidad Libre con un $16.8 \%$ y la Santiago de Cali, con el $12.8 \%$.
} 
(a) No hubo

matrícula

en

este

año.

LO

$\mathrm{s}$ admitidos iniciaron clases en febrero, 1980.

El ingreso a la Universidad del Valle supone, entonces, un proceso previo de selección social que elimina competidores pero que no suprime las dificultades de la competencia ya que esta última no se define únicamente en términos del volumen de participantes, sino también en relación con ciertas características sociales que vale la pena entrar a examinar. En efecto, los aspirantes a la Universidad poseen unos rasgos definidos, mas o menos constantes ${ }^{21}$ se trata de una población constituida principalmente por hombres; es demográficamente joven y, en el caso de los hombres, cada vez más joven; no más de uno de cada seis aspirantes trabaja; procede de colegios oficiales más que de privados, localizados casi en su totalidad en el Valle del Cauca, especialmente en Cali y Palmira; con una formación clásica y, en proporción menor, técnica y con un rendimiento aceptable en el bachillerato. Quiere ello decir que los aspirantes de sexo femenino, los mayores de 20 años, aquellos que se ven, por diferentes circunstancias, obligados a combinar estudio y trabajo y los que no poseen las características educativas mencionadas tienen una menor probabilidad de aspirar a un cupo en la Universidad del Valle que sus similares con rasgos opuestos.

Tomando en cuenta este perfil, podría concluirse que aparentemente la Universidad del Valle continúa, como en el pasado, siendo un establecimiento reservado a unos cuantos privilegiados. Esta afirmación encierra algo Ce verdad en la medida en que sólo unos pocos pueden llegar a la educación superior, a juzgar por lo que señalan las tasas de escolarización; sin embargo, es un hecho que la Universidad ha dejado de acoger el mismo tipo de estudiante de hace diez o veinte años. En realidad, la diferenciación interna del sistema universitario nacional y regional ${ }^{22}$ y la llegada de nuevas clases y capas sociales a la educación secundaria han transformado de manera fundamental la composición social de los aspirantes a la Universidad del Valle. La característica más notable de ese cambio ha sido la mayor participación proporcional, en el conjunto de aspirantes y estudiantes, de jóvenes que provienen de las clases medias asalariadas y de los sectores "populares" (empleados no calificados y obreros) de la región y de la ciudad. Los datos del Cuadro 2, aunque limitados en su intención comparativa ${ }^{23}$, debido a la ausencia de información sobre aspirantes a la Universidad hace 10 ¿15 años, reflejan sin

\footnotetext{
${ }^{21}$ Los datos están tomados de una encuesta realizada a los aspirantes a la Universidad en 1978 y que hace parte del estudio mencionado atrás sobre Aspirantes y Estudiantes de la institución.

${ }^{22}$ Entiéndese por diferenciación interna el proceso mediante el cual el crecimiento del sistema universitario se lleva a cabo mediante la consolidación de dos subsistemas, la universidad pública y la universidad privada, cada uno con rasgos y tendencias propios. Para el caso colombiano, consúltese el trabajo de Rama, así como el más reciente de Franco y Tunnermann, La Educación Superior de Colombia, FES, Bogotá, 1978. Igualmente, el lúcido ensayo de Gerardo Molina, "Universidad Estatal y Universidad Privada" publicado en la recopilación ¿Universidad Oficial o Universidad Privada? Tercer Mundo, Bogotá, 1978. Sobre el Valle del Cauca, aún no existe un estudio a fondo de las tendencias del sistema universitario regional. Pueden, sin embargo, consultarse algunas publicaciones de la Oficina de Planeación y Desarrollo de la Universidad del Valle.

${ }^{23}$ Obsérvese que se está comparando dos poblaciones hasta cierto punto, diferentes (aspirantes y estudiantes) de universidades distintas y a partir de un indicador que abarca sólo de manera parcial el concepto de "origen social" de los aspirantes. No obstante, las poblaciones comparadas tienen en común su relación con la Universidad como estudiantes potenciales o efectivos; de otra parte, como el mismo Rama lo reconoce, la Universidad Nacional es representativa de la educación superior colombiana y puede configurar un buen punto de referencia para el estudio de otros establecimientos oficiales. Finalmente, aunque el indicador empleado es muy limitado, diferencia sin embargo a los asalariados de los no asalariados y establece categorías dentro de cada uno de esos sectores. No es ni mucho menos un indicador de clase social (las clases no se "miden" mediante "indicadores"), aunque puede proporcionar una pista —eso sí, bastante modesta— en esa línea de análisis.
} 
embargo, una tendencia más o menos transparente. En efecto, la comparación de las dos distribuciones señala un cambio en el origen social. Mientras en 1967 el peso de los propietarios era mayoritario (58.6\%), en el caso de los aspirantes de 1978 esos sectores no alcanzan a representar la mitad de la población. En contrapartida, el grupo de asalariados medios y, sobre todo, el de sectores obreros y de empleados no calificados aumentaron considerablemente. Se aprecia, entonces, una mayor participación de aspirantes de origen popular, lo cual, de todas formas, no puede ocultar la presencia aún cuantitativamente importante de los aspirantes provenientes de familias propietarias.

¿Qué alcance posee, entonces, la afirmación de que la Universidad colombiana es una universidad de las "clases medias"? Afirma Rama que "la Universidad Nacional se caracteriza por el predominio de los sectores medios en la composición de su matrícula. Su peso es de casi un $80 \%$ del total (...) El nivel medio superior y el nivel medio medio están representados, cada uno de ellos con el $28 \%$ de la matrícula y el medio inferior con el $22 \%{ }^{24}$. Cataño adhiere a esa interpretación: “... los estudiantes de la Universidad Nacional provienen en su mayoría, no de las 'clases económicamente menos favorecidas' del Rector Patiño ni de las 'familias en extremo pobres' del Profesor Gerardo Molina, sino de la clase inedia, en cuyo seno el volumen más considerable pertenece a los estratos medios medios y medios altos" 25 .

A juzgar por los datos, estas opiniones se encuentran un poco alejadas de la realidad. Sin embargo, buena parte del asunto parece radicar en la conceptualización empleada. Tanto Rama como Cataño utilizan el término de "clases medias" como sinónimo de estratos sociales, construidos mediante la agrupación de categorías ocupacionales con un nivel similar de prestigio social y con rasgos más o menos compartidos en términos de ingresos y niveles de consumo. Así, en el estrato superior Rama incluye a los patrones de más de 50 empleados, los profesionales independientes, los altos jefes y los rentistas. Cataño, por su parte, incluye en la clase alta a los patrones con más de 50 empleados, altos jefes de la administración pública y privada y rentistas. Aunque desde el punto de vista del manejo de la información el concepto de estrato así definido facilita las cosas y permite ciertas descripciones, es evidente que, por su carácter eminentemente clasificatorio, no sólo menoscaba la posibilidad de interpretación sociológica sino que, además, da pie a una manipulación de los datos por parte del investigador. En el caso de Rama, por ejemplo, la clasificación, tal y como fue elaborada, conduce necesariamente a la conclusión de que los estudiantes de la Universidad Nacional proceden de las "clases medias". Al fin de cuentas, de cinco hay tres estratos (60\%) que conforman esas "clases".

\footnotetext{
${ }^{24}$ Rama, Germán W., op. cit. p. 80.

${ }^{25}$ Castaño. Gonzalo, op. cit. p. 220.

Digitalizado por RED ACADEMICA
} 


\section{CUADRO 2}

\begin{tabular}{|c|c|c|c|c|}
\hline \multicolumn{5}{|c|}{$\begin{array}{c}\text { CATEGORIA OCUPACIONAL DEL PADRE DE LOS ASPIRANTES A LA } \\
\text { UNIVERSIDAD DEL VALLE (1978) Y DE LOS ESTUDIANTES DE LA UNIVERSIDAD } \\
\text { NACIONAL (1967) - (\%) }\end{array}$} \\
\hline \multirow{2}{*}{$\begin{array}{l}\text { Categoría ocupacional } \\
\text { Grandes propietarios }\end{array}$} & \multicolumn{2}{|c|}{$\begin{array}{c}\text { Aspirantes } \\
\text { Univalle(1978) }\end{array}$} & \multicolumn{2}{|c|}{$\begin{array}{l}\text { Estudiantes U. } \\
\text { nacional (1967) }\end{array}$} \\
\hline & 10.6 & & 15.4 & \\
\hline Patrón con mas de 50 empleados & & 1.2 & & 2.3 \\
\hline Patrón con 6 a 40 empleados & & 3.5 & & 11.5 \\
\hline Rentista & & 5.9 & & 1.6 \\
\hline Pequeños propietarios & 37.5 & & 43.2 & \\
\hline Patrón con 1 a 5 empleados & & 9.9 & & 13.8 \\
\hline Trabajador independiente con local & & 11.9 & & 9.5 \\
\hline Trabajador independiente sin local & & 10.8 & & 14.5 \\
\hline Profesional independiente & & 4.9 & & 5.4 \\
\hline Asalariados medios & 30.3 & & 26.5 & \\
\hline Alto jefe & & 3.6 & & 4.8 \\
\hline Jefe inmediato & & 8.0 & & 8.3 \\
\hline Pequeño jefe & & 7.9 & & 4.8 \\
\hline Empleado de calif. Tecn. o Univ. & & 10.8 & & 8.6 \\
\hline Populares & 20.8 & & 14.9 & \\
\hline Empleados no calificados & & 9.1 & & 7.7 \\
\hline Obreros calificados & & 8.9 & & 4.4 \\
\hline Obreros no calificados & & 2.8 & & 2.5 \\
\hline Capataces & & & & 0.3 \\
\hline Otros & 0.8 & & & \\
\hline total & 100.0 & & 100.0 & \\
\hline
\end{tabular}

Fuentes: Encuesta a Aspirantes (1978), Univalle.

Rama, Germán W., op. cit. p. 79.

Si los criterios de organización de la información son aquellos que han sido utilizados en el Cuadro 2, la interpretación tiene que variar. En efecto, allí se consideran "capas medias" al grupo identificado como "asalariados medios" y que comprende, desde el punto de vista de su definición teórica, a todos aquellos asalariados que trabajan realizando una labor de carácter eminentemente intelectual; poseen, además, funciones de control y mando que implican no sólo tareas propias sino posiciones de jerarquía y autoridad. Si se asume esa óptica, resulta claro que la universidad pública en Colombia no es ni ha sido la universidad de las "clases medias". En 1967, en la Universidad Nacional los estudiantes provenían básicamente de sectores propietarios (58.6\%), siendo minoritaria la representación de asalariados medios y, mucho más, de sectores de origen popular. En el caso de la Universidad del Valle, si bien el peso de los propietarios ha disminuido, sigue siendo significativo, a la par que se incrementa la participación de aspirantes de origen social diferente (asalariados medios y populares).

La visión de la Universidad como "feudo" de las clases medias ha sido, entonces, otro de tantos mitos con los que hemos convivido desde hace años pero que, como todo mito, encierra más fantasía que realidad.

Dos aspectos de importancia se desprenden de la interpretación anterior: en primer lugar, lo que actualmente ocurre en la Universidad del Valle, en términos de los cambios 
en la composición social de sus aspirantes refleja uno de los rasgos más marcados del sistema universitario del país, a saber, el de la correspondencia entre diferenciación interna del sistema universitario (universidad pública/universidad privada) y "especialización" social de cada uno de esos tipos de universidad. Los establecimientos públicos tienden cada vez más a acoger a un estudiantado de origen popular y de capas medidas asalariadas, aunque el sector de pequeños propietarios posee aún una representación no despreciable, mientras que el sector privado responde prioritariamente a una demanda de grandes y medianos propietarios y de estratos altos de pequeños propietarios y de asalariados medios.

En segundo lugar, no obstante los cambios señalados, la Universidad del Valle, y posiblemente la Universidad colombiana, continúa siendo "la imagen invertida de la sociedad", tal como lo señaló Rama ${ }^{26}$. Al confrontar la categoría ocupacional del padre de los aspirantes con la distribución de la de los ocupados en Cali, en 1978, resulta que hay sobre-representación de los propietarios y del grupo de asalariados medios, y subrepresentación de los obreros y empleados no calificados. Estos constituyen el 55.3\% de los ocupados en Cali, mientras que en la Universidad representan solamente una quinta parte de los aspirantes. De nuevo saltan a la vista las desigualdades ante la Universidad, esta vez en términos de las posibilidades de aspirar a ser estudiante, mayores para unos grupos sociales, menores para otros.

Pero, la competencia por un cupo en la Universidad del Valle no sólo es difícil sino desigual. Teniendo en cuenta que quienes pretenden llegar a la Universidad constituyen un grupo heterogéneo socialmente y diferenciado internamente en cuanto a sus medios y modos de reproducción social, es lógico pensar que cada sector enfrenta las pruebas de admisión en condiciones dispares pues, según sea su origen social, los aspirantes van a estar mejor o peor provistos de las armas necesarias para sortear con éxito los exámenes. Estas ramas no se refieren únicamente a las habilidades o capacidades intelectuales de cada individuo sino también al bagaje académico-científico que ha logrado acumular hasta el momento, al ambiente cultural que lo ha rodeado, a la existencia de condiciones (materiales, sicológicas, familiares, etc.) para el aprendizaje, en fin, a la preparación lograda para la prueba de admisión.

Ahora bien, lo que primero sorprende al examinar la información sobre probabilidades de ingreso a la Universidad (Ver Cuadro 3) es que, en el nivel de las categorías gruesas, existe una relación inversa entre origen social de la población de aspirantes y probabilidad de "pasar" la prueba de admisión. Son, en efecto, los aspirantes de origen popular los que muestran una mayor probabilidad de admisión ${ }^{27}$ mientras que los jóvenes que proceden de los sectores de propietarios se ubican por debajo del promedio ponderado.

¿Cómo entender este paradójico resultado? Podría pensarse que las familias de altos ingresos, considerando la educación de sus hijos como una inversión económica, envían a los mejor dotados a las universidades privadas puesto que así aseguran una rápida y segura recuperación de la inversión. La universidad pública estaría acogiendo a los estudiantes de menor rendimiento académico de esas familias, razón por la cual sus probabilidades de admisión resultan inferiores a las de sus similares de procedencia social diferente. Esta hipótesis, que puede ser denominada hipótesis de la "racionalidad económica" aunque llamativa, no parece muy convincente pues supone que los aspirantes de bajo nivel académico tienen cabida en la Universidad del Valle. Y ello no ocurre así. En

\footnotetext{
${ }^{26}$ Rama, Germán W., op. cit. p. 83.

${ }^{27}$ Como puede verse, las probabilidades de admisión están medidas por la relación admitidos/aspirantes (RAA), que indica la proporción de aspirantes que superaron exitosamente los exámenes de admisión.
} 
efecto, la admisión a la Universidad del Valle supone una cierta calidad académica: de una parte, se exige un puntaje mínimo en el examen del ICFES, superior en algunos casos al exigido por otros centros de enseñanza superior; de otra parte, las pruebas de admisión contienen un grado de dificultad no despreciable, mayor que el de los exámenes de otras universidades, por lo que se requiere una buena preparación previa. Finalmente, y a pesar de las opiniones parcializadas de los enemigos de la Universidad, se sabe que la mayoría de los planes de estudio de la Universidad del Valle son académicamente exigentes y precisan generalmente una dedicación de tiempo completo por parte del estudiante.

CUADRO 3

\begin{tabular}{|c|c|c|}
\hline \multicolumn{3}{|c|}{$\begin{array}{l}\text { RELACION ADMITIDOSIASPIRANTES SEGÚN EL ORIGEN SOCIAL DE LOS } \\
\text { ASPIRANTES A LA UNIVERSIDAD DEL VALLE - } 1978\end{array}$} \\
\hline Categorías agregadas & Promedio ponderado & Categorías desagregadas \\
\hline & & $\begin{array}{l}.353 \text { Empleados de calif. Tecn. } \\
\text { Univ. }\end{array}$ \\
\hline & & .336 Empleados no calificados \\
\hline & & $\begin{array}{ll}.321 & \text { Patrón de } 1 \text { a } 5 \\
& \text { empleados }\end{array}$ \\
\hline & & $.320 \quad$ Alto jefe \\
\hline & & 311 Pequeño jefe \\
\hline & & Obrero calificado \\
\hline Populares .319 & & Profesional independiente \\
\hline \multirow[t]{2}{*}{ Asalariados medios .316} & & Obrero no calificado \\
\hline & 296 & \\
\hline Pequeños propietarios .290 & & $\begin{array}{l}.294 \text { Patrón con mas de } 150 \\
\text { empleados }\end{array}$ \\
\hline \multirow[t]{4}{*}{ Grandes propietarios .215} & & 278 Trabajador indep. Con local \\
\hline & & 269 Jefe inmediato \\
\hline & & $\begin{array}{ll}212 & \text { Patrón con } 6 \text { a } 49 \\
\text { empleados }\end{array}$ \\
\hline & & 177 Rentista \\
\hline
\end{tabular}

Fuente: $\quad$ Encuesta a Aspirantes, 1978 (Univalle)

No obstante, la hipótesis de la racionalidad económica contiene un elemento cuyo análisis aporta la clave para comprender el problema. En efecto, dicha hipótesis se funda en el fenómeno de la diferenciación interna del sistema universitario y su relación con la composición social de la población estudiantil. Hablar de universidad privada significa pensar en altos precios de matrícula y, por tanto, en un "filtro" de selección de estudiantes, de carácter económico. Quienes desean cursar estudios en establecimientos privados deben, por tanto, poseer los medios para pagar matrículas elevadas y demás gastos necesarios para su subsistencia (libros, mantenimiento mensual, etc.). Quienes no se hallen en esa condición, en general aspirantes de origen popular o provenientes de sectores medios asalariados de ingresos medios y bajos, deben competir por los cupos que ofrece la Universidad del Valle, donde los niveles de matrícula son sustancialmente inferiores. Es esa la única forma posible de seguir una carrera universitaria. No tienen otra alternativa, a no ser la de olvidarse temporal o definitivamente de ingresar a la Universidad. Su tabla de salvación consiste, entonces, en garantizar un aceptable nivel 
académico o, por lo menos, una adecuada preparación para el examen de admisión, no sólo porque la preparación adquirida durante el bachillerato no les asegura necesariamente el éxito, sino porque deben competir con aspirantes que, por su origen social, han logrado una mejor formación académica y se presentan "mejor armados" al examen y, por lo tanto, con una mayor probabilidad teórica de ser admitidos.

Si los aspirantes que provienen de familias de recursos medios o bajos muestran una mayor probabilidad de admisión es porque, en la medida de sus posibilidades, se han preparado a fondo para el examen de admisión con la única finalidad de aprobarlo. Así se entiende que una alta proporción de bachilleres, según sus disponibilidades de tiempo y recursos, se matriculen en cursos "pre-universitarios" que se llevan a cabo generalmente durante la noche y los fines de semana, destinados a preparar al aspirante única y exclusivamente para enfrentar el examen de ingreso a la Universidad.

Por su parte, los bachilleres de familias de altos ingresos tienen un abanico de posibilidades un poco más amplio: su formación en bachillerato, recibida por lo regular en colegios de alto nivel académico, les proporciona los elementos suficientes para superar las admisiones en la Universidad del Valle. Si no ocurre así, pueden matricularse en cursos pre-universitarios, cuyo costo no representa mucho para sus familias, y lograr la preparación necesaria. Y si ello no basta, no tienen la alternativa en el caso de varias profesiones $^{28}$, de acudir a la Universidad privada.

En estas condiciones, la competencia se vuelve desigual no sólo porque el aspirante de altos ingresos tiene la posibilidad de elegir entre varias universidades, ni únicamente porque el bagaje cultural que ha logrado acumular hasta el momento lo provee de una mejor calificación para enfrentar el examen de admisión, sino, además, porque no se halla "forzado", como sí lo están los aspirantes de bajos ingresos, a invertir un volumen adicional de tiempo, recursos y esfuerzos para obtener aquellos conocimientos que no pudieron acopiar durante el bachillerato - independientemente de lo que hayan querido o no- y que aparecen como requisito indispensable para ingresar a la universidad.

El hecho de que los aspirantes de origen popular tengan una mayor probabilidad de ingresar a la Universidad, ¿significa que la hipótesis de la selección social no tiene mucho que ver en la explicación de las tendencias actuales? No parece ser el caso. Lo que ocurre es que el mecanismo de selección opera de otra forma: en primer lugar, diferenciando la población que va a establecimientos privados de la que sólo puede aspirar a un cupo en la universidad pública; en segundo lugar, haciendo posible para unos y difícil o imposible para otros (los que no disponen de tiempo y recursos) matricularse en cursos pre-universitarios.

Lo que sí queda claro es que el examen de admisión, en contra de lo que alguna vez se llegó a pensar, tiene un peso innegable en la escogencia de los estudiantes. No es el factor fundamental de selección, según se ha visto, pero tiene que ver en el éxito o fracaso de los aspirantes. Al fin de cuentas, se convierte en uno de tantos obstáculos -reales- que el aspirante debe superar y para lo cual debe realizar un entrenamiento. Sin embargo, su eficacia se encuentra ligada a los procesos de selección social que operan previamente y que encuentran en él su último peldaño. Como ya ha sido demostrado $^{29}$, el examen de admisión no es una prueba "igual" para todos los aspirantes

\footnotetext{
${ }^{28}$ Las carreras en que compiten la Universidad del Valle y las privadas son Medicina. Economía, Administración y Contaduría. algunas ramas de Ingeniería y varias Licenciaturas de Educación.

${ }^{29}$ Ver el trabajo de Lugardo Alvarez y Jaime Niño, Las clases Sociales y la Admisión a la Universidad, monografía de licenciatura. Universidad Nacional, Bogotá, 1969, (Mimeo). 
ya que sus contenidos suponen unos conocimientos, un lenguaje y unas capacidades que no todos los bachilleres están en condición de certificar y que se corresponden con el tipo de colegio, el ambiente cultural familiar y las posibilidades que tiene el aspirante de entrar en contacto con distintas formas y canales de conocimiento, por fuera de la escuela, todo lo cual es función de su condición social ${ }^{30}$.

\section{Las preferencias académicas: La Universidad y los procesos de selección social.}

La interpretación ofrecida hasta aquí no deja de ser parcial e incompleta; incluso, puede contener algo de error en la medida en que ha tomado como base el análisis de datos agregados, globales, sin tener en cuenta las diferencias que pueden presentarse en las distintas áreas académicas de la Universidad. El examen de estas últimas puede, en efecto, proporcionar un cierto grado de precisión a las conclusiones hasta aquí esbozadas, razón por la cual vale la pena detenerse en ese aspecto.

La distribución de la demanda por cupos en las distintas áreas académicas de la Universidad del Valle señala la permanencia de una misma tendencia en los últimos años, definida por la concentración de solicitudes en los programas de Salud e Ingeniería y, de manera secundaria, de Ciencias Sociales y Económicas. Las dos primeras especialidades atrajeron en 1981 dos de cada tres solicitudes y, en conjunto, las tres áreas concentraron el $80 \%$ de la demanda. El resto de programas, especialmente los de Educación, atendieron un bajo volumen de inscripciones. En consecuencia, la competencia por cupos no es la misma en las distintas carreras, sino que se vuelve más difícil en unas que en otras.

Este proceso de diferenciación adquiere rasgos más definidos al comparar los niveles de la demanda con la oferta de cupos por parte de la Universidad (Cuadro 4). No sólo la competencia es más nutrida en algunas áreas, concretamente Salud, Arquitectura e Ingeniería, sino que los aumentos más significativos en los niveles de competencia se registran precisamente en ellas.

\footnotetext{
${ }^{30}$ La selección que opera por la vía del origen social es la fundamental, pero no la única. En realidad, se halla reforzada por otros canales selectivos, imposibles de examinar dentro de los límites de este trabajo. La encuesta a los aspirantes mostró que las probabilidades de ingreso a la Universidad son mayores para los hombres que para las mujeres, para los más jóvenes y los más "adultos" que para los de edad intermedia; para los que ya han presentado alguna vez el examen de admisión que para los que lo hacen por primera vez; para quienes han tenido un mejor rendimiento en el bachillerato que para los que han repetido años en secundaria. Así, el sexo, la edad, la "experiencia en la presentación de exámenes y algunas características educativas constituyen mecanismos complementarios de selección que refuerzan los procesos básicos ya examinados.
} 


\section{CUADRO 4}

\begin{tabular}{|l|c|c|}
\hline \multicolumn{3}{|c|}{$\begin{array}{c}\text { COMPETENCIA POR CUPOS EN LA UNIVERSIDAD DEL VALLE } \\
\mathbf{1 9 7 3} \text { Y } \\
\mathbf{1 9 8 1}\end{array}$} \\
\hline Áreas académicas & $\mathbf{1 9 7 3}^{*}$ & $\mathbf{1 9 8 1}$ \\
\hline Ciencias & 2.3 & 2.1 \\
\hline Humanidades & 1.3 & 1.6 \\
\hline Ciencias sociales y económicas & 3.8 & 4.4 \\
\hline Educación & 0.9 & 1.0 \\
\hline Arquitectura & 3.0 & 5.2 \\
\hline Salud & 5.2 & 15.3 \\
\hline Ingeniería & 4.1 & 6.8 \\
\hline Total & 3.2 & 5.3 \\
\hline
\end{tabular}

Fuente: Univalle, Oficina de Planeación y Desarrollo.

* La cifra indica el número promedio de aspirantes por cupo ofrecido.

Tal evidencia permite delimitar con mayor precisión dos grandes grupos de especialidades: de un lado, áreas con altos niveles de competencia (Salud, Ingeniería y Arquitectura); de otro, áreas con un bajo nivel de competencia (Educación, Humanidades, Ciencias y, de manera relativa, Ciencias Sociales y Económicas) ${ }^{31}$.

Las diferencias entre estos dos tipos de especialidades no son meramente cuantitativas sino que se refieren a sus propiedades específicas, lo cual explica los grados diversos de concentración de la demanda. En primer lugar, las profesiones del primer grupo poseen, a diferencia de las del segundo, un mercado de trabajo con una demanda potencial superior a la oferta de profesionales. En consecuencia, las perspectivas futuras que presentan estas especialidades al aspirante le garantizan una mínima seguridad contra el desempleo.

En segundo lugar, esas especialidades prometen al estudiante un nivel promedio de ingresos superior al de otras profesiones, por lo menos como tendencia, ya que en cada rama es posible encontrar una distribución heterogénea de los ingresos. De todos modos, en la óptica de las expectativas, la dimensión del ingreso esperado incide indudablemente en las preferencias de los aspirantes y, por tanto, en los niveles de competencia en cada área. Esta propiedad reviste importancia en relación con dos factores: de un lado, el deterioro progresivo del poder de compra de los colombianos, especialmente los asalariados; de otro, las apetencias de ascenso social y de acceso a niveles superiores de consumo, posibilitadas en parte por un determinado monto de ingresos.

En tercer lugar, en el primer grupo hay predominio de las llamadas profesiones "liberales" que pueden ser ejercidas de manera independiente y, por tanto, no se hallan sometidas necesariamente al marco de la relación salarial. Con las especialidades del segundo grupo sucede algo diferente pues su ejercicio no es posible por fuera de ese marco. Esto significa ingresos fijos, ubicados, generalmente, en niveles intermedios de la escala y sujetos a un directo control fiscal y al progresivo deterioro de su poder adquisitivo.

\footnotetext{
${ }^{31}$ El caso de Ciencias Sociales y Económicas ha sida colocado en este grupo, a pesar de que muestra mayores niveles de competencia en comparación con las restantes áreas del grupo. Esos mayores niveles se explican por la creciente demanda de una de sus ramas, la Administración. Si se hace caso omiso de esta última, el resto de ramas permanecen en un bajo nivel de competencia por cupos, muy lejos de las disciplinas del primer grupo.
} 
Una cuarta diferencia radica en los niveles de prestigio social, definido como la importancia social otorgada a las distintas profesiones, con los privilegios que de allí se desprenden. Esa importancia puede ser referida a varios factores: calidades académicas que se consideran necesarias para cada especialidad; papel que puede desempeñar en el agenciamiento del cambio social; capacidad de vinculación con el aparato político; correspondencia con una determinada condición social, etc. Es indudable que por las transformaciones de todo orden, incluso ideológicas, que se han presentado en nuestro medio, el factor prestigio ha ido perdiendo terreno dentro del conjunto de criterios que orientan la preferencia del aspirante; sin embargo, también es cierto que ese elemento constituye todavía un punto de referencia primordial en la decisión y que, en el caso de profesiones como Arquitectura y de las ramas de Salud, sigue desempeñando un papel importante.

Por último, los dos grupos de especialidades suponen un "perfil estudiantil" distinto. Las altamente competidas exigen por lo general un estudiante de tiempo completo pues la carga académica lo obliga a permanecer la mayor parte del tiempo en la Universidad y le impone un trabajo por fuera de la clase y de la Universidad que copa su jornada diaria y le impide, por ejemplo, combinar estudio y trabajo remunerado. Por otra parte, esas mismas carreras son más "costosas" en términos de los "insumos" requeridos (libros, instrumental, implementos de trabajo, etc.), de modo que el estudiante —o su familia- debe contar con una cierta solvencia económica y con condiciones propicias para responder a las exigencias académicas.

Todas estas diferencias, más otras que no han sido enumeradas, son evidentemente de carácter social y hacen que la aspiración del bachiller adquiera visos diferentes según se trate de un tipo de especialidad o de otro. Más aún, esas diferencias se corresponden con el origen social de los aspirantes que tiende a predominar en cada una de las áreas académicas, haciendo ver con más claridad el fenómeno de la competencia. En efecto, como puede verse en los datos del Cuadro 5, la distribución de los aspirantes según su origen social no es la misma para cada especialidad. De hecho, puede establecerse una tipología de áreas académicas con cuya ayuda será posible interpretar con mayor exactitud el problema de la competencia. Los tipos más relevantes son los siguientes:

\section{CUADRO 5}

\begin{tabular}{|c|c|c|c|c|c|c|c|c|}
\hline \multicolumn{9}{|c|}{$\begin{array}{l}\text { UNIVALLE-RELACION ADMITIDOS/ ASPIRANTES EN LAS DISTINTAS AREAS ACADEMICAS SEGÚN } \\
\text { ORIGEN SOCIAL DE LOS ASPIRANTES } 1978\end{array}$} \\
\hline $\begin{array}{l}\text { Categoría } \\
\text { ocupacional } \\
\text { del padre }\end{array}$ & Ciencias & Humanidades & economía & educación & arquitectura & Salud & ingeniería & total(a) \\
\hline $\begin{array}{l}\text { Grandes } \\
\text { propietarios }\end{array}$ & .400 & .571 & .500 & & .500 & .141 & .156 & .233 \\
\hline $\begin{array}{l}\text { Pequeños } \\
\text { propietarios }\end{array}$ & .564 & .566 & .364 & .375 & .372 & 3165 & .300 & .290 \\
\hline $\begin{array}{l}\text { Asalariados } \\
\text { medios }\end{array}$ & .548 & .596 & .495 & .286 & .397 & .185 & .318 & .316 \\
\hline Populares & .536 & .523 & .387 & .294 & .367 & .130 & .356 & .319 \\
\hline Otros & & 1.000 & .400 & & & .100 & .571 & .333 \\
\hline Toral & .544 & .566 & .414 & .286 & .385 & .164 & .316 & .302 \\
\hline
\end{tabular}

Fuente: $\quad$ Encuesta a Aspirantes, 1978.

(a) Los datos totales correspondientes a las categorías ocupacionales del padre no concuerdan con los del Cuadro 2, pues ha sido suprimida la categoría de "Rentistas".

Tipo A: Áreas con un predominio de aspirantes procedentes de familias de pequeños y grandes propietarios y de estratos altos de asalariados medios: Salud y Arquitectura. 
Tipo B: Áreas con un peso significativo de aspirantes de origen popular: Educación.

Tipo C: Áreas socialmente heterogéneas, como las anteriores, pero que no señalan el predominio de ningún grupo social en especial. Pueden distinguirse dos subtipos:

C1: Áreas con leve tendencia a acoger más aspirantes de sectores medios y de pequeños propietarios que de otro origen: Ingeniería y Humanidades.

C2: Áreas con un peso relativamente mayor de aspirantes de origen popular y de capas de asalariados medios: Ciencias y Economía.

Esta tipología corresponde en términos generales a la que se venía manejando hasta ahora, sólo que es más precisa en cuanto señala con detalle las diferencias entre especialidades con respecto al origen social de sus aspirantes. En esa medida, permite un análisis más fecundo, sobre todo porque se basa en el criterio del origen social, fundamental para cualquier interpretación sociológica. Pero, lo más importante de la tipología es que ayuda a entender los niveles diferenciales de dificultad de la competencia por cupos para los distintos grupos de aspirantes. En efecto, se aprecia que existe una correspondencia entre niveles de competencia y composición social de la población de aspirantes en una determinada especialidad: a mayor competencia, mayor la tendencia a que los aspirantes del área procedan de familias propietarias o pertenecientes a los estratos altos de los grupos asalariados medios. Esta correspondencia no es casual. En realidad, las especialidades caracterizadas por un alto grado de competencia suponen, como ya se señaló, un cierto "tipo ideal" de estudiante cuyos rasgos centrales son los de solvencia económica, posibilidad de dedicarse exclusivamente al estudio y cierto nivel académico y cultural. Este tipo de aspirante tiene mayor probabilidad de pertenecer a familias cuyas condiciones de reproducción hacen posible ese conjunto de rasgos y que son, en general, familias de altos ingresos y con amplias oportunidades de acceso a los diferentes canales culturales.

Para un aspirante de origen popular, entonces, la competencia por el ingreso a la Universidad resulta difícil no sólo porque debe sobreponerse a una serie de barreras que existen en los niveles primario y secundario de la educación, ni únicamente porque cada vez encuentra más bachilleres luchando por el mismo cupo que él, ni tan sólo porque los aspirantes con los que compite tienen un origen social diferente y se hallan mejor provistos para ganar el examen, sino además porque su origen social constituye hasta cierto punto un obstáculo para aspirar a determinadas profesiones que la misma sociedad se ha encargado de convertir en reserva para ciertos grupos sociales.

Esta conclusión plantea de inmediato la cuestión de las probabilidades de admisión en cada especialidad, según el origen social. ¿Sucede en cada especialidad lo que ha sido descrito para la Universidad en su conjunto, o existen ciertas especificidades que hacen que en algunas áreas académicas los mecanismos selectivos operen con más fuerza? 


\section{CUADRO 5A}

\begin{tabular}{|l|c|c|c|c|c|c|c|c|}
\hline \multicolumn{7}{|c|}{ UNIVALLE- ORIGEN SOCIAL DE LOS ASPIRANTES EN LAS DISTINTAS AREAS ACADEMICAS 1978 (\%) } \\
\hline categoría ocupacional del padre & ciencias & \multicolumn{1}{c|}{$\begin{array}{l}\text { humanidad } \\
\text { es }\end{array}$} & economía & educación & arquitectura & salud & ingeniería & total \\
\hline Grandes propietarios (b) & 4.4 & 4.0 & 3.7 & 8.6 & 4.9 & 6.5 & 4.0 & 5.1 \\
\hline Patrón con 50 o mas empleados & & 0.6 & 1.2 & & 1.0 & 2.2 & 0.6 & 1.3 \\
\hline Patrón con 6 a empleados & 4.4 & 3.4 & 2.5 & 8.6 & 3.9 & 4.3 & 3.4 & 3.8 \\
\hline Pequeños propietarios & 34.2 & 43.4 & 37.0 & 22.9 & 41.9 & 41.1 & 39.4 & 39.7 \\
\hline Patrón con 1 a 5 empleados & 8.8 & 10.3 & 8.1 & 2.9 & 16.6 & 10.0 & 11.1 & 10.5 \\
\hline Trab. Independiente con local & 9.6 & 12.6 & 12.9 & 5.7 & 8.8 & 13.1 & 13.8 & 12.6 \\
\hline Trab. Independiente sin local & 14.9 & 15.4 & 13.2 & 14.3 & 10.2 & 9.9 & 11.3 & 11.4 \\
\hline Profesional independiente & 0.9 & 5.1 & 2.8 & & 6.3 & 8.1 & 3.2 & 5.2 \\
\hline Asalariados medios & 36.8 & 26.9 & 28.5 & 20.0 & 38.1 & 35.0 & 29.8 & 32.2 \\
\hline Alto jefe & 6.1 & 3.4 & 2.8 & 5.7 & 5.4 & 3.9 & 3.2 & 3.8 \\
\hline Jefe intermedio & 7.9 & 6.9 & 7.2 & 2.9 & 8.8 & 10.4 & 7.4 & 8.5 \\
\hline Pequeño jefe & 11.4 & 8.0 & 7.5 & 5.7 & 7.8 & 8.1 & 9.1 & 8.4 \\
\hline Emp. De col. Tecn. o Univ. & 11.4 & 8.6 & 11.0 & 5.7 & 16.1 & 12.6 & 10.1 & 11.5 \\
\hline Populares & 24.6 & 25.1 & 29.2 & 48.5 & 14.6 & 16.4 & 25.9 & 22.1 \\
\hline Empleados no calificados & 11.4 & 10.3 & 9.7 & 11.4 & 5.8 & 9.5 & 10.4 & 9.7 \\
\hline Obreros calificados & 8.8 & 10.8 & 13.2 & 25.7 & 7.3 & 5.7 & 12.3 & 9.5 \\
\hline Obreros no calificados & 4.4 & 4.0 & 6.3 & 11.4 & 1.5 & 1.2 & 3.2 & 2.9 \\
\hline Otros & & 0.6 & 1.6 & & 0.5 & 1.0 & 0.9 & 0.9 \\
\hline & 100. & 100.0 & 100.0 & 100.0 & 100.0 & 100.0 & 100.0 & 100.0 \\
\hline
\end{tabular}

(b) no incluye "Rentistas"

Fuente: Encuestas a aspirantes, 1978. 
La hipótesis que se desprende del análisis de la tipología sugiere que en las especialidades de Tipo A la RAA es baja para los aspirantes de origen popular y mayor para los aspirantes de sectores medios y propietarios; por su parte, en las especialidades Tipo $\mathrm{B}$, los aspirantes de sectores populares tendrían una probabilidad de ingreso más cercana a la de los aspirantes de origen social diferente. En cuanto a los subtipos $\mathrm{C} 1 \mathrm{y}$ $\mathrm{C} 2$, podría pensarse que la tendencia en el primero sería similar a la del Tipo $A$ y, en el segundo, cercana al Tipo B. En el Cuadro $6^{32}$ está consignada la información respectiva. ¿Qué señalan los datos?

La hipótesis es correcta para las áreas de Tipo A y Tipo B. Los datos sobre las especialidades Tipo C1 confirman la hipótesis para Humanidades, pero no para Ingeniería. En realidad, este último caso es sui generis pues es la única área en que la mayor RAA corresponde a los aspirantes de origen popular y la menor a los hijos de familias propietarias. Aunque no se cuenta con la información empírica necesaria para explicar esta situación, puede afirmarse que cabe la interpretación sugerida en la segunda sección de este trabajo a propósito de los datos agregados para la Universidad. Teniendo presente que ésta es una especialidad con una proporción considerable de aspirantes de origen popular, no resulta arriesgado afirmar que para ellos y, en general, para la mayoría de los aspirantes a esas carreras la única posibilidad de cursarlas es ingresando a la Universidad del Valle no sólo porque ofrece programas que no existen en otros establecimientos, o porque la universidad garantiza una buena formación, sino porque las matrículas son baratas. En consecuencia, quienes poseen el talento necesario (buenos conocimientos en matemáticas y ciencias exactas) o tienen la posibilidad de prepararse adecuadamente para el examen de admisión, logran una mayor probabilidad de ingreso. Por lo demás, este tipo de disciplinas técnicas no exigen cierta clase de pre-requisitos (conocimientos generales, culturales, ambiente intelectual, etc.) que sí son fundamentales en otras especialidades, por ejemplo, Arquitectura, Humanidades y Ciencias Sociales.

Finalmente, en lo que respecta a las carreras Tipo C2, los datos no parecen dar fundamento empírico a la hipótesis, pues las mayores probabilidades de ingreso están del lado de los sectores medios asalariados y de los aspirantes que provienen de familias propietarias.

\footnotetext{
${ }^{32}$ Se han tomado las categorías agregadas pues señalan más claramente las tendencias pertinentes para el análisis.
} 
CUADRO 6

\begin{tabular}{|c|c|c|c|c|c|c|c|c|c|c|c|}
\hline \multicolumn{12}{|c|}{$\begin{array}{c}\text { RELACION ADMITIDOSIASAPIRANTES POR CATEGORIA OCUPACIONAL DEL PADRE DEL ASPIRANTE SEGÚN AREA } \\
\text { ACADEMICA- UNIVERSIDAD DEL VALLE, } 1978\end{array}$} \\
\hline \multirow{2}{*}{$\begin{array}{c}\text { Categoría } \\
\text { ocupacional del } \\
\text { padre }\end{array}$} & \multicolumn{3}{|c|}{ Tipo A } & \multicolumn{3}{|c|}{ Tipo c1 } & \multirow{2}{*}{$\frac{\text { Tipo B }}{\text { Educación }}$} & \multicolumn{4}{|c|}{ Tipo c2 } \\
\hline & Arquitectura & Salud & Total & Ingeniería & Humanidades & Total & & Ciencias & Economía & Total & TOTAL \\
\hline $\begin{array}{c}\text { grandes } \\
\text { propietarios }\end{array}$ & .500 & .141 & .189 & .156 & .571 & .231 & & .400 & .500 & .471 & .233 \\
\hline $\begin{array}{l}\text { pequeños } \\
\text { propietarios }\end{array}$ & .372 & .165 & .201 & .300 & .566 & .351 & .375 & .564 & .364 & .414 & .290 \\
\hline $\begin{array}{l}\text { asalariados } \\
\text { medios }\end{array}$ & .397 & .185 & .224 & .318 & .596 & .364 & .286 & .548 & .495 & .511 & .316 \\
\hline populares & .367 & .130 & .167 & .356 & .523 & .385 & .294 & .536 & .387 & .421 & .319 \\
\hline total & .385 & .164 & .202 & .316 & .566 & .359 & .286 & .544 & .414 & .449 & .302 \\
\hline
\end{tabular}

Fuente: Encuestas a aspirantes, 1978. 
El panorama de las distintas áreas académicas deja, entonces apreciar que, como tendencia general, las mayores probabilidades de ingreso a la Universidad del Valle recaen sobre los sectores medios asalariados y propietarios y no sobre los sectores populares, con la única excepción de Ingeniería. Si se recuerda el análisis de la sección anterior, estos resultados parecen contradecir las conclusiones allí logradas. En efecto, se señaló, tomando como base la información agregada, que los aspirantes de origen popular y de capas medias asalariadas tenían mayor probabilidad de ingreso a la Universidad que el resto de aspirantes. Ese fenómeno fue explicado como el efecto de un esfuerzo adicional realizado por los aspirantes de bajos ingresos para adquirir la preparación adecuada que los capacitara para la prueba de admisión. Este esfuerzo adicional —-se decía - refleja una situación de desigualdad, máxime si se tiene en cuenta que muchos aspirantes no disponen de los medios necesarios para realizarlo y que los hijos de familias acomodadas tienen ante sí una gama mucho más extensa de opciones de estudio.

En realidad, no se trata de una incoherencia o contradicción en el análisis. Lo que hacen los datos del Cuadro 6, por el contrario, es precisar y delimitar el verdadero alcance de esas conclusiones. Si la información agregada mostraba que los aspirantes de origen popular tenían más probabilidades de ingreso, era porque esa probabilidad estaba determinada por los resultados del área de Ingeniería en la que ese tipo de aspirantes representa un gran volumen (35.70/o de los aspirantes de origen popular en toda la Universidad) y poseen la I\{AA más alta dentro del área. Se trata, entonces, de un caso de ponderación del promedio. Los datos de Ingeniería determinan el comportamiento de los datos globales y aclaran la imagen imprecisa creada por estos últimos. Al analizar la información por áreas académicas queda claro lo que realmente ocurre. En el caso de Ingeniería, la interpretación formulada en la sección anterior es adecuada. Para las demás especialidades, las cifras muestran que los aspirantes de origen popular se encuentran en desventaja en términos de probabilidades de ingreso y que son los sectores medios asalariados y de grandes propietarios los que salen mejor librados del examen. En todas las áreas, la RAA de los asalariados medios se encuentra por encima del promedio del área respectiva.

Ahora bien, lo interesante de este nuevo resultado es que el peso creciente de los aspirantes que proceden de los sectores medios asalariados se ve reforzado por una mayor probabilidad de admisión en las distintas arcas. Esto lleva a pensar que, en una perspectiva de mediano o largo plazo, de continuar las actuales tendencias, la Universidad del Valle, y posiblemente la universidad pública en general, será la universidad de las clases medias. Es verdad que los sectores de obreros y empleados no calificados también vienen ejerciendo una fuerte presión sobre los cupos de la Universidad; sin embargo, sus bajas probabilidades de ingreso impedirán que estos grupos pasen a constituir una porción mayoritaria de la población estudiantil. Con respecto a los aspirantes de altos ingresos la situación es más compleja pues su capacidad de elegir entre universidad pública o privada obliga a pensar en otros factores (niveles académicos, diversificación de programas, costo de los estudios, estabilidad institucional, etc.) para saber hasta dónde seguirán ejerciendo presión sobre los cupos de la universidad pública. Lo cierto es que la expansión progresiva de los establecimientos privados en la región ha vuelto más transparente la "especialización social" de cada subsistema universitario, transformando en consecuencia la composición social de la población estudiantil en la universidad pública.

Se completa de esta manera el cuadro general de los procesos de selección social. Los canales selectivos son muy variados y tienen que ver, de un lado, con factores que operan 
mucho antes del ingreso a la Universidad y que determinan quiénes pueden aspirar a la educación superior y quiénes no; de otro, con procesos relacionados con la admisión, los cuales señalan un conjunto de probabilidades de ingreso al grueso de estudiantes, diferentes según su origen social y el tipo de área académica escogida.

Una de las formas más directas de expresión de los procesos selectivos es la relación entre origen social y probabilidades de admisión en ciertas áreas académicas. Más concretamente, se trata de las profesiones clasificadas como Tipo A (Salud y Arquitectura) en las que los aspirantes de familias de altos ingresos muestran un nivel de admisión mucho más alto que sus similares de origen social diferente. Esta evidencia señala la estrecha ligazón entre procesos de selección y reproducción social y, más precisamente, la medida en que los mecanismos selectivos se convierten en instrumentos de reproducción social.

Hasta el momento, los autores que se han referido al tema de la educación como instrumento de reproducción social han colocado el acento en el mismo tipo de generalidades. Cito tan sólo dos ejemplos entre muchos: Vasconi ${ }^{33}$ afirma que la escuela capitalista ha sido una creación histórica de la burguesía en el poder, que cumple una doble función de reproducción de la sociedad burguesa y de sus modalidades de explotación: capacita mano de obra para satisfacer las demandas del aparato productivo; impone y difunde las formas ideológicas y culturales dominantes. Baudelot y Establet ${ }^{34}$ expresan una opinión similar, aunque en forma más compleja, mostrando cómo la escuela capitalista consagra la separación entre trabajo manual y trabajo intelectual mediante la diferenciación de dos redes de escolarización, y cómo esto se convierte en una forma de reproducción de las relaciones de dominación.

En verdad, la educación, y en este caso la Universidad, cumple esa doble función. Al fin de cuentas, capacita y habilita a los estudiantes para el ejercicio de una especialidad y posibilita mediante múltiples mecanismos la transmisión de concepciones valorativas y de prácticas ideológicas. La Universidad no es una isla y se halla atravesada e, incluso, determinada por un contexto social, por una lógica de organización y funcionamiento de las relaciones sociales, de la cual hace parte y de cuyos traumas y vicisitudes no puede escapar. Sin embargo, el análisis de los aspirantes a la Universidad del Valle ha permitido visualizar un nuevo elemento, tanto o más importante, que los anteriores. En efecto, no solamente la Universidad califica cuadros e inculca ideologías sino que consagra diferencias sociales por la vía de la "especialización social" de sus distintas áreas académicas. Aspirar a ciertas profesiones y, sobre todo, ingresar a la Universidad para estudiarlas supone ciertos requisitos sociales que, en caso de no ser cumplidos, producen una situación de amplia desventaja para los "no elegidos". Por su parte, esas profesiones permiten consolidar una cierta condición social de los estudiantes, con todos los privilegios sociales que se derivan de ella, y ahonda en esa medida las diferencias sociales entre los estudiantes y, posteriormente, en la sociedad. Las otras especialidades, particularmente las catalogadas en los tipos B y C2, acogen a estudiantes que no entran en el círculo de los "elegidos" 35 , pero contribuyen igualmente a la reproducción social en la medida en que consagran la condición social de "no privilegiado".

\footnotetext{
${ }^{33}$ Vasconi, Tomás, Contra la Escuela, Ediciones Hombre Nuevo, Medellín, (sin fecha).

${ }^{34}$ Ver la Introducción de este trabajo.

${ }^{35}$ Los términos "elegidos" y "no elegidos" no tienen aquí el mismo significado que les otorgan Bourdieu y Passeron. Los empleo para describir a dos grandes sectores estudiantiles que se diferencian por el tipo de carrera a la que pueden aspirar, en función de su diverso origen social.
} 
Sucede, entonces, dentro de la Universidad algo así como lo que señalan Baudelot y Establet para la escuela capitalista en Francia. Hay dos "redes", dos vías, dos canales de formación universitaria que, si bien comparten el hecho de pertenecer a una misma institución, se diferencian en sus pre-requisitos sociales, en sus contenidos académicos y culturales, en sus formas de funcionamiento y, muy seguramente, en el tipo de prácticas estudiantiles que las caracterizan. Tales "redes" no aluden, claro está, como en Baudelot y Establet, a la separación trabajo manual-trabajo intelectual, sino que, dentro del sector de los trabajadores intelectuales, señalan y consagran diferencias sociales que se corresponden con algunas de las existentes en la sociedad. Los estudiantes que pasan por esas dos redes son objeto de un determinado "entrenamiento", no solamente académico sino también social, mediante el cual se hace posible posteriormente su localización en la división social del trabajo. De esta manera, la Universidad contribuye a la estructuración no de una clase sino de un conjunto de relaciones sociales que inciden en el conjunto de relaciones de clase en la sociedad. La población estudiantil es entonces heterogénea no solamente en relación con su "Origen social", como lo señalan Bourdieu y Passeron, sino también con respecto a lo que podría llamarse su "destino" social, es decir, su futura inserción en una lógica de relaciones sociales. Esa inserción les otorga un lugar de privilegio dentro de la sociedad, en su calidad de profesionales; pero ese privilegio no es el mismo para todos sino que se halla diferenciado según el tipo de profesión y, por tanto, según la clase de "entrenamiento" recibido.

Este proceso de "asignación" de especialidades según la pertenencia social del aspirante no opera, sin embargo, de una manera ineluctable e irreversible. Pensarlo así sería aceptar una especie de fatalismo y, por tanto, trasladar a la sociología la teoría de la predestinación. Así lo han hecho algunas corrientes de pensamiento, en especial ese estructuralismo que mira a la sociedad como un conjunto de estructuras que se mueven ciegamente mediante causas "objetivas", sobre las cuales el individuo no tiene capacidad de maniobrar ni posibilidad de ejercer algún tipo de influencia. Las prácticas sociales son vistas como "efectos" de la interrelación de estructuras y no como producto de sujetos que actúan en condiciones determinadas. El estructuralismo y todas aquellas corrientes que miran a la sociedad de esa manera son interpretaciones que matan al hombre y, por tanto, a la historia pues la convierten en un simple resultado de procesos que "se dan" y de los cuales los sujetos no son más que soportes, para no decir que títeres.

Por el contrario, es preciso mirar las cosas con otro lente. En efecto, si bien existen procesos objetivos que se desarrollan hasta cierto punto con independencia de la voluntad de sujetos históricos y que llegan a ser condicionantes, incluso limitantes, de la acción de los individuos, también es cierto que la vida social sería algo incomprensible si no se acude a la observación y al análisis de lo que los individuos son, piensan y hacen. La existencia de dos canales de entrenamiento social dentro de la Universidad, entre los cuales se distribuye la población de estudiantes, es un hecho social inocultable. Sin embargo, si bien esa distribución se lleva a cabo en función del origen social, no puede negarse que los aspirantes cuentan con una capacidad de decisión y, por tanto, con la posibilidad de "quebrar" esa ley objetiva de asignación de los lugares dentro de la Universidad. No de otra manera puede interpretarse la heterogeneidad social de la población de aspirantes y estudiantes en las diferentes áreas académicas y, más exactamente, la participación de aspirantes de origen popular en carreras de tipo A que no son las que "teóricamente" les corresponden por su origen social. Sólo en esa óptica pueden ser entendidos los mayores grados de competencia en unas áreas que en otras y el desarrollo de esfuerzos adicionales de los aspirantes para obtener un cupo en dichas áreas. 
Este fenómeno puede ser examinado como una búsqueda de ascenso social por parte de los aspirantes de origen popular y de capas bajas de los grupos medios asalariados, o como un medio de acceder a grados más elevados de bienestar o, inclusive, corno una manera de asegurar en el futuro un nivel de ingresos que garanticen protección contra las dificultades económicas que ya están afrontando ciertas capas de profesionales universitarios. Cualquiera que sea la interpretación, ese fenómeno deja ver que los procesos de reproducción social no se llevan a cabo sin obstáculos y que los mecanismos de reproducción operan al lado de otros mecanismos que van en dirección contraria. La lógica de funcionamiento de una sociedad es, entonces, el resultado de la tensión entre esos dos tipos de mecanismos. En otras palabras, es un proceso de reproducción/negación que tiene sus efectos sobre la Universidad. Es precisamente esa dialéctica la que asigna rasgos específicos a la vida universitaria y al papel de la Universidad con respecto a la sociedad. En medio de esa tensión, o como parte componente de ella, las prácticas sociales de los individuos, en este caso los aspirantes, tienen mucho que ver en la reproducción de una lógica social o en su reestructuración. Y es por ello que no se las puede olvidar en el análisis de la institución universitaria.

Cali, julio de 1982 